\title{
Turnover of sex defining mutation provides an insight into evolution of sex chromosomes in the golden pompano (Trachinotus ovatus)
}

\author{
Liang Guo ${ }^{1}$, Jing-Wen Yang ${ }^{2}$, Baosuo Liu $^{2}$, Danilo Malara ${ }^{2}$, Pietro Battaglia ${ }^{2}$, Khor \\ Waiho $^{2}$, Nan Zhang ${ }^{2}$, Kechneg Zhu ${ }^{2}$, Huangyang $\mathrm{Guo}^{2}$, Bo Liu ${ }^{2}$, Fang-Cao Zhao', \\ Dianchang Zhang ${ }^{3}$, and Shigui Jiang ${ }^{2}$ \\ ${ }^{1}$ State Key Laboratory of Biocontrol, Institute of Aquatic Economic Animals and the \\ Guangdong Province Key Laboratory for Aquatic Economic Animals \\ ${ }^{2}$ Affiliation not available \\ ${ }^{3}$ South China Sea Fisheries Research Institute, Chinese Academy of Fishery Sciences
}

November 16, 2021

\begin{abstract}
The golden pompano (Trachinotus ovatus) is a marine fish species in the family Carangidae. We constructed a chromosome-level genome assembly of a male golden pompano. QTL-mapping and GWAS analysis showed that this species has a ZZ/ZW sex determination system and a sex defining SNP (Chr16:18219150:G/A), located on the splice donor site (GT-AG) of the first intron of Hsd17b1, was exclusively associated with the phenotypic sex. The W-linked coding sequences of Hsd17b1 were conserved across vertebrates, while Z-linked coding sequences introduced extra 64 bases and were malfunctional. The golden pompano and the greater amberjack (Seriola dumerili), divergent in 57 million years ago in the same family, share the same features of sex determination, including the same sex determining gene, malfunctional Z-linked haplotypes, undifferentiated sex chromosomes except that the sex defining SNPs are different. In simulation analysis, turnover of sex determining mutation, single mutation dominating sex determination and undifferentiated sex chromosomes were also observed. We proposed a hypothesis that Wlinked haplotypes of the sex determining gene of Hsd17b1 were under purifying selection, Z-linked haplotypes may evolve near neutrally, recurrent and directional transformations from W-linked haplotypes to Z-linked haplotypes caused by inactivating mutations, relatively strong forces of drift and recombination comprehensively contributed to turnover of sex defining mutation and undifferentiation of sex chromosome. We also established zebrafish mutants and homozygous mutants were "all male", which indirectly supported this hypothesis.
\end{abstract}

\section{INTRODUCTION}

Sex determining genes refer to genes that are expressed in undifferentiated gonad and directly determine the bipotential gonad into either a testis or an ovary (Capel, 2017; Mei, Gui, 2015). It is just three decades ago that Sry was confirmed as the first sex determining gene via the creation of a transgenic XX mouse carryingSry (Koopman et al. , 1991). Based on the discovered sex determining genes, it was postulated that new master sex determining gene originates from either gene duplication followed by sub- or neofunctionalization, or allelic diversification (Kikuchi, Hamaguchi, 2013). For example, the sex determining gene $S R Y$ in human was proposed to arise from SOX3 in the form of allelic diversification (Graves, 2015).

Dozens of sex-determining genes have been identified in vertebrates (Taoet al. , 2021), and these genes are not conserved in amphibia (Miura, 2018), reptiles (Modi, Crews, 2005) and fish (Volff, 2002), from orders, families and genera to species. From an evolutionary perspective, teleosts branched out from lobe-finned fish and tetrapod lineages with a third genome duplication occurred in the stem lineage of teleosts (Froese, Pauly, 2011). In quantitative terms, teleosts constitute the dominant radiation of vertebrates and also has 
the highest diversity of genetic sex determination systems in vertebrates (Mank, Avise, 2009). Among the confirmed sex determining genes in teleosts (Tao et al. , 2021),BCAR1 in Ictalurus punctatus (Bao et al. , 2019),dmrt1 in Cynoglossus semilaevis (Chen et al. , 2014), Amhr2 in Takifugu rubripes (Kamiya et al. , 2012), and Gsdf $f^{Y}$ in Oryzias luzonensis (Myosho et al. , 2012) are assumed to arise through allelic diversification. The fate of known master sex determining genes has only been explored in medakas (Myosho et al. , 2015), salmonids (Guiguen et al. , 2018) and the order of Esociformes (Pan et al. , 2021).

Canonical researches on sex chromosomes are based on the linkages of birds and mammals, they are featured with high differentiation between the $\mathrm{X}$ and $\mathrm{Y}$ or $\mathrm{Z}$ and $\mathrm{W}$ chromosomes and degeneration of $\mathrm{Y}$ or $\mathrm{W}$ chromosome, respectively (Wright et al. , 2016). The widely prevailing hypothesis of sexual antagonistic selection is that selection would occur within a region between a sex-determining gene and a nearby gene with sex-specific effects, thereby eventually leads to recombination suppression and heteromorphic chromosome (Ponnikaset al. , 2018). In contrast, many lineages of fish, amphibians, and non-avian reptiles have only slightly differentiated sex chromosomes. In $90 \%$ of gonochoristic fish species studied, the sex chromosomes are visibly homomorphic based on karyotypes (Devlin, Nagahama, 2002). Two models have been proposed to explain their homomorphy, namely the high turnover model (Schartl, 2004; Volffet al. , 2007) and the Fountain-of-Youth model (Perrin, 2009). The high turnover model is based on the idea that turnover may occur frequently, so that, even if recombination is suppressed, sex chromosomes are replaced by different chromosomes before they have time to degenerate, as documented in African cichlids, true frogs, flies, medaka fishes and African clawed frogs (Ponnikas et al. , 2018). The Fountain-of-Youth model points that a long-term differentiation is prevented by occasional XY (or ZW) recombination in sexually reversed individuals $(0.1 \%$ is far enough in simulations), in which the arrest of XY recombination in males is controlled by maleness per se , as documented in common frogs (Grossen et al. , 2012; Rodrigueset al. , 2018). It seems that recombination suppression is inevitable based on the observed cases and the phenomenon that the sex chromosomes are undifferentiated except the sex-linked mutation, at the other extreme of sex chromosome divergence, is rare in metazoa but independently evolved in the greater amberjack (Seriola dumerili) (Koyama et al. , 2019) and the tiger pufferfish (Takifugu rupripes ) (Kamiya et al. , 2012). In these two species different sex-determining genes, Hsd17b1 in the greater amberjack and Amhr2 in the tiger pufferfish, arose through allelic divergence and both act in a dominate fashion, ZZ/ZW and XX/XY, respectively. The tiger pufferfish shares the same sex-determining gene and the sex-determining mutation with the panther puffer (T. pardalis ) and the finepatterned puffer (T. poecilonotus ) (Kamiyaet al. , 2012), which empirically hints that the undifferentiation of the sex chromosomes in the tiger pufferfish persisted for at least 4.7 million years since their divergence. The greater amberjack also shares the same sex-determining gene and mutation with closely related species, the yellowtail amberjack (S. lalandi ) and the Japanese amberjack (S. quinqueradiata ) (Koyama et al. , 2019). Recently, a neutral model was proposed to emphasize the role of purely neutral forces on the initiation and inhibition of recombination in sex chromosome evolution, however, the fact that sex determining gene was under selection was left out (Jeffries et al. , 2021).

In the yellowtail amberjack, the sex defining SNP (SNP1196) in the third exon of the sex determining gene $H s d 17 b 1$ introduces a missense mutation (G->E) in Z-linked protein and leads to $99.66 \%$ reduction of catalytic activity (Koyama et al. , 2019). The genus Seriola belongs to the subfamily Naucratinae and the clade (Naucratinae, Caranginae) in the family Carangidae. The golden pompano (Trachinotus ovatus ) belongs to the subfamily Trachinotinae of the same family and the clade (Scomberoidinae, Trachinotinae) (SupFig 1 and Figure 5 ). We constructed a high-quality chromosome-level male (ZZ) genome assembly and characterized the sex-determining system of the golden pompano. Interestingly, undifferentiated sex chromosomes were also observed and the sex-determining mechanism was assumed to be the same between the yellowtail amberjack and the golden pompano, including the sex-determining gene and malfunction of Z-linked haplotypes, except that the sex defining SNPs are different. Based on the neutral model and the sex-determining mechanism, we proposed a hypothesis to explain the turnover of sex determining mutation and undifferentiation of sex chromosomes. Simulation analysis was also performed and the results supported this hypothesis. We also established zebrafish mutants to indirectly supports that Hsd1 $7 b 1$ in the golden pompano and the greater amberjack may be not necessary in male development. 


\section{MATERIALS AND METHODS}

\subsection{Genome sequencing and assembly}

DNA was isolated from a male T. ovatus . A 40-kb library was constructed and sequenced on the PacBio SeuqelII platform (Pacific Biosciences, CA, USA). A 300-bp paired-end Illumina fragment library was constructed and sequenced on the NovaSeq platform (Illumina, CA, USA). RNA was extracted from the gill, brain, heart, liver, spleen, kidney, stomach, intestines, skin, fin, muscle, testis and ovary. Equal amounts of RNA from each tissue were mixed to generate one sample. An Iso-seq library was prepared and sequenced on two cells of a PacBio Sequel system (PacBio, CA, USA). Sequences were processed using SmartLink 5.0 software to obtain full-length and non-chimaeric consensus (FLNC) sequences for gene prediction. In addition, RNA-Seq data from eight tissues, including muscle, liver, spleen, kidney, stomach, skin, brain, gill, gonad and intestines and Hi-C data, both sequenced on the Illumina platform, were obtained from a previous study (Zhang et al. , 2019).

Genome size and heterozygosity were estimated with Jellyfish v2.2.10 (Marçais, Kingsford, 2011) and GenomeScope v1.0 (Vurture et al. , 2017) with $84 \mathrm{M}$ reads ( $40 \times$ ). In total, 6.5 M PacBio subreads were obtained with a minimum length of $500 \mathrm{bp}$. The median-length subreads were subjected to assembly with the Falcon toolkit v1.8.1 (Chin et al. , 2016). The primary contigs (Tov-G1) were polished with PacBio long reads using Arrow v2.3.3 (Chin et al. , 2013) and Illumina short reads using Pilon v1.23 (Walker et al. , 2014). The polished contigs were retrieved from the NCBI nucleotide database v5 with blastn (Altschul et al. , 1997) to remove contaminants. A total of $382 \mathrm{M} H \mathrm{Hi} C \mathrm{C}$ paired-end read pairs were used to anchor the contigs into scaffolds (Tov-G2). These reads were mapped to the contigs with Juicer (Durand et al. , 2016). The chromosome-level assembly was obtained with a 3D-DNA pipeline (Matthewset al. , 2018). The assembly was manually curated with Juicebox v1.11.01 (Durand et al. , 2016). The gaps in the pseudochromosomes were filled with PBJelly v15.8.24 (English et al. , 2012). Then, the gap-filled scaffolds were polished again as above.

The completeness of the final genome assembly was assessed using BUSCO v4.0.5 with actinopterygii_odb10 (Sim O et al. , 2015). The quality of each base in the final assembly was calculated with Referee v1.1.2 (Thomas, Hahn, 2019). The quality of the final assembly was also assessed with the alignment rate. The median-length PacBio genome reads were mapped with minimap2 v2.17 ( $\mathrm{Li}, 2016)$. The FLNC sequences were mapped with GMAP v2020-06-30 (Wu, Watanabe, 2005). RNA-Seq reads were mapped with HISAT2 v2.1.0 (Kim et al., 2015).

\subsection{Assembly validation with a genetic linkage map}

Re-sequencing has been performed for a full-sib family (F201803) in a previous study, including two parents and 100 offspring (Guo et al. , 2020). The genetic linkage map was constructed as previously (Guo et al. , 2019). The optimal LOD score was selected with an overall consideration of the marker distribution in each linkage group, allocation stability and group number (SupFig $\mathbf{3 b}$ ). The haplotype chromosome number is $24 \mathrm{inT}$. ovatus (Hu et al. , 2007). Finally, the linkage groups were assigned an LOD score of 14 . The marker order in each linkage group was obtained according to the best score from 10 independent runs. Genetic distance was converted by using the Kosambi mapping function. The positions of SNPs on the genetic linkage map and assembly were compared as Pearson correlation coefficient.

\subsection{Genome Annotation}

Transposable elements were detected and classified using RepeatMasker v4.0.9 and the Dfam_3.0 database (Hubley et al. , 2016), in which the query species was set to Teleostei. Gene structure was predicted using MAKER v2.31.10 (Cantarel et al. , 2008). The first round was run to predict gene models from transcript evidence and protein evidence with an ab initio predictor, Augustus v3.3.3 (Stanke et al. , 2006). The transcript evidence included FLNC sequences and the RNA-seq transcript assembly. RNA-seq reads were mapped to the final genome assembly with HISAT2 v2.1.0 (Kim et al. , 2015), and the transcript assembly was predicted with StringTie v2.0.3 (Pertea et al. , 2016) by using the conservative model (-conservative). The 
protein evidence included the proteins from the uniprot_sprot database (Apweiler et al. , 2004). The program Augustus was trained with conserved genes in BUSCO. The second round was run to predict gene models from transcript evidence and protein evidence with ab initio predictors, namely, SNAP v2006-07-28 (Korf, 2004) and Augustus v3.3.3 (Stanke et al. , 2006). SNAP was trained with complete gene models surrounded by genomic sequences obtained in the first round with AED set to 0.5. The program Augustus was trained with the predicted proteins in the first round. The descriptive statistics of gene models were obtained for the primary transcripts by Eval v2.2.8 (Keibler, Brent, 2003). Functional annotation was performed with eggNOG-mapper v2 (Huerta-Cepaset al. , 2017), which was based on orthology assignments. Noncoding RNAs, including rRNAs, snRNAs, miRNAs, and tRNAs, were identified using Infernal v1.1.2 (Kolbe, Eddy, 2009) with the Rfam database 14.1 (Kalvari et al. , 2018).

\subsection{Estimation of the divergent time between $T$. ovatusand $S$. dumerili}

In addition to T. ovatus, the protein sequences of other 14 fish species, namely, Astyanax mexicanus, Danio rerio ,Gasterosteus aculeatus, Larimichthys crocea, Lates calcarifer, Seriola dumerili, Lepisosteus oculatus ,Mola mola, Oreochromis niloticus, Oryzias latipes ,Poecilia formosa, Takifugu rubripes, Tetraodon nigroviridis and Xiphophorus maculatus, were downloaded from the Ensembl database 2014 (Flicek et al. , 2013) and included for phylogenetic analysis. Primary coding sequences and protein sequences of each gene were extracted. Protein sequences in their corresponding coding sequences containing termination codons or unknown bases were excluded (SupTab 14 ). Gene families were identified using OrthoFinder2 (Emms, Kelly, 2019). Protein sequences of the single-copy genes were aligned with MAFFT v7.450 in auto mode (Kuraku et al. , 2013), and the alignment of corresponding nucleotide sequences was obtained with PAL2NAL v14 (Suyama et al. , 2006). A phylogenetic tree was constructed using IQ-TREE v1.6.2 (Nguyen et al. , 2015) with extended model selection and 1000 ultrafast bootstraps. The GTR $+\mathrm{F}+\mathrm{R} 3$ model was selected according to the Bayesian information criterion. The divergence time between species was estimated using MCMCTree in PAML version 4.9j (Yang, 2007). Two calibration time points based on fossil records (takifugu-tetraodon: 32.25 56.0 Ma; zebrafish-medaka: 149.85 165.2 Ma) (Benton, Donoghue, 2007; Yang et al. , 2016) were integrated, and the age of the root was constrained as the origin of modern ray-finned fishes (approximately $385 \mathrm{Ma}$ ) (Giles et al. , 2017). The prior for the substitution per site per year was set to $10^{-8}$. The independent rates model was selected, and convergence was validated by comparing the posterior mean times between repeated analyses (Dos Reis, Yang, 2019). The time tree was visualized with MCMCTreeR (Puttick, 2019).

\subsection{Investigation of the sex ratio in families}

A mass-cross population of T. ovatus (PM2018), which contained 1726 offspring, has been assigned into families with SSR markers in a previous study (Guo et al. , 2020). When these offspring grew up to 2 years old, 1083 individuals were sampled, weighed, and sexed by dissection and and gonad observation. The ovary and testis were distinguished by their appearance (Jiang et al. , 2015). The number of female and male in each family was counted and the hypothesis that the number of female is equal to the number of male in each full-sib family was tested with Chi-square test. The hypothesis that there is no difference of body weight between sex and family was tested by Analysis of Variance. The hypothesis tests were performed with IBM SPSS statistics version 20 (IBM SPSS INC, Chicago).

\subsection{Detection of sex QTL}

The sex QTL was detected with methods of QTL mapping and GWAS. The full-sib family (F201803 ) used to construct the genetic map were also included in the mass-cross population (PM2018). The 905 discordant SNPs in the genetic map and the assembly were removed. The remaining SNPs within each group were reordered with 20 repeats using Lep-MAP3 (Rastas, 2017) and the best order with highest likelihood were processed as the final genetic map. The phased genotypes and female and male averaged map were exported as F2 population. The sex QTL were detected with MapQTL 6 (Ooijen et al., 2009). The potential QTL were first detected using internal mapping, and the threshold of the significant level of the LOD score was determined using a permutation test with a $p$ value of 0.05 and with 10,000 permutations. Then, the SNP closest to the significant QTL was taken as a cofactor to narrow the interval in subsequent multiple 
QTL mapping.

82 individuals (37 males and 45 females) collected from natural population were subjected to paired-end $(2 \times 150 \mathrm{bp})$ re-sequencing on Illumina platform. Their sex identification and SNPs calling were performed as above. The association between sex and genotypes was detected with genotypic test using PLINK v1.90b6.2 (Purcell et al. , 2007). The whole genome significant $p$ value was set to $10^{-10}$. The closely related species in the family Carangidae, including eight "T. ovatus" (Mediterranean), 15T. blochii, one Caranx ignobilis and one C. melampygus, was used in checking the change of focused SNPs. The samples of T. ovatus (Mediterranean) was collected from Mediterranean and the $15 \mathrm{~T}$. blochii were collected from the local farm. The data of C. ignobilis and C. melampygus was downloaded from SRA of NCBI. The reads were mapped to the genome assembly as above. The focused SNPs (Chr16:18219150 and Chr16:18219853) were checked with the text alignment viewer in SAMtools v1.9 (Liet al. , 2009).

\subsection{The features of sex chromosomes}

Simple repeat was detected using TRF v4.09 (Benson, 1999) with default settings. The percentage of bases in the simple repeats was scanned in non-overlapped 10-kb windows. The read depth in the two parents of the full-sib family was also scanned in non-overlapped 10-kb windows and compared as log2(male/(female+0.1)). With the genetic map and physical map constructed, the local recombination rates of female were calculated using MareyMap v1.3 (Rezvoy et al. , 2007) with Loess based method. The nucleotide diversity in the natural population was estimated in 10kb non-overlapped window using VCFtools v0.1.16 (Danecek et al., 2011). The linkage disequilibrium in the natural population was estimated using LDBlockShow v1.36 (Dong et al. , 2021).

\subsection{Validation of alternative splicing}

The RNA from a one-year ovaries and a one-year testes were subject to long read sequencing and RNA-seq, respectively. Two other sets of short reads from ovary and testes were downloaded from SRA with the age unavailable. For PacBio Iso-seq data processing, we used the Iso-seq3 pipeline (Gonzalez-Garay, 2015) to construct FLNC transcripts. The transcript from Hsd17b1 was identified by mapping the FLNC transcripts to the genome assembly using Minimap2 (Li, 2018). The transcripts were constructed with StringTie v2.1.4 (Kovaka et al. , 2019). The short reads from Illumina platform were mapped to the genome assembly using HISAT2 (Kim et al. , 2019) with mapQ above 30. The transcript expression was quantified with Ballgown (Frazeeet al. , 2015; Pertea et al. , 2016). Splicing events within the gene region of Hsd17b1 were visualized as a Sashimi plot in Integrative Genomics Viewer (Thorvaldsdóttir et al. , 2013).

\subsection{The alignment of the protein sequences of $H S D 17 B 1$}

Protein isoforms of $H s d 17 b 1$ were obtained with ExPASy translate tool (Gasteiger et al. , 2003). The protein sequences of HSD17B1 from vertebrate were collected and aligned with MEGA X and ESPript v3.0 (Robert, Gouet, 2014). The phylogenetic tree was constructed as neighbor-joining tree with Hsd17b9 and Hsd17b7 as outgroup. The robustness was tested with 1000 bootstraps. The three dimensional structure was predicted using SWISS-MODEL (Waterhouse et al. , 2018) with human HSD17B1(ID: 1iol.1) as a template, which was complexed with 17 beta-estradiol (Azzi et al. , 1996).

\subsection{Simulations of evolution of Hsd17b1}

Simulation analysis of the evolution of Hsd17b1 (1678 bp in length, including exons and introns) was performed with the sex-determining SNP (Chr16:18219250) existed in the initial population. In each nonoverlapping generation, neutral point mutations were allocated at random positions at rate $\mu$. Recombination occurs at a rate of $\mathrm{r}=\mathrm{r}_{0} \times(1-3 \% \times \mathrm{d})$, in which $\mathrm{r}_{0}$ was set as the local recombination rate in female of the mapping family, $d$ was the divergence between haplotypes and the coefficient (3\%) was referring to the parameters in the neutral model (Jeffries et al. , 2021). Missense mutations in exons led to the transformation from $\mathrm{W}$ haplotype to $\mathrm{Z}$ haplotype, as well as base changing in the alternative splicing sites. Population size $(\mathrm{N})$ was limited to $25,50,100,200$. Three combinations of recombination rate $\left(\mathrm{r}_{0}\right)$ and mutation rate $(\mu)$ were used to examine the influence of population size, i.e., $1 \times 10^{-6}$ and $1 \times 10^{-8}, 1 \times 10^{-4}$ and $1 \times 10^{-6}, 1 \times 10^{-2}$ 
and $1 \times 10^{-4}$, respectively (Table 2 ). Simulations were run for 20,000 generations, and the average number of nucleotide differences between haplotypes $(\pi)$ and the average number of nucleotide differences between haplotypes from two sex chromosome pools $\left(\mathrm{d}_{\mathrm{xy}}\right)$ were used to assess the processes (Nei, Li, 1979). The associations between population size and nucleotide diversity and differences were tested with $\mathrm{R}$ package: basicTrendline (Mei W, 2018)

\subsection{Establishment of knockout lines of zebrafish}

The AB strain zebrafish obtained from CZRC (China Zebrafish Resource Center, Wuhan, China) was used for gene knockout. CRISPR/Cas9 target sites were designed using an online tool ZiFiT Targeter software (http://zifit.partners.org/ZiFiT), which identified sequence 5'GG-(N18)-NGG3' in the first exon (CCTTGCTGTGCATCTCGCATCAA), the second exon (CTATGCTACTATGCGGAACTTGG) and the third exon (AGGACCCATAAGACCCACAC and GGACACTATAAGAGCCATCC) of Hsd1 $7 b 1$ in zebrafish (ID: ZDB-GENE-040901-5, ZFIN). Each sgRNA was synthesized by cloning the annealed oligonucleotides into the sgRNA expression vector pT7-gRNA followed by in vitro transcription. pSP6-2sNLS-spCas9 plasmid was linearized by XbaI and capped Cas9 mRNA was synthesized by T7mMESSAGE Ultra@Kit (Ambion). The concentrations of the capped mRNAs were measured by NanoDrop (Thermo Scientific, Waltham, MA) and its quality was examined by agarose gel electrophoresis. Microinjection was performed on the zebrafish embryos at one cell stage . 100 pg sgRNA and 600 pg Cas9 RNA were co-injected. Non-injected embryos were used as controls. All embryos were maintained in an environmental incubator at $28 \mathrm{degC}$ for at least one day before viability examination. The male and female F1 fish with the same frame shift mutations were crossed to produce homozygous F2 mutants (-/-). The mutation was confirmed by directing Sanger sequencing.

\subsection{Influence of the mutation of $H s d 17 b 1$ in zebrafish}

Fish were sexed based on secondary features (Romano et al. , 2020) and confirmed with hematoxylin-eosin staining on gonads on day 145 post fertilization. Male fertility of the homozygous F2 mutants was assessed by mating performance, which was defined according to their ability to stimulate spawning of the wild-type females. At least two male fish of each genotype $(+/+$ or $-/-)$ were tested with the wild-type $(+/+)$ female fish separately. The tests were repeated at 5-day intervals. The spawning rate was defined as the ratio of successful spawning pairs over total pair number after 24 hours. The experiment was repeated at least three times.

\section{RESULTS}

\subsection{A high-quality genome assembly of $T$. ovatus was constructed}

PacBio sequencing produced 6.72 M subreads (>500 bp,SupTab 1 and SupTab 2 ). From each polymerase read, the median-length subread was extracted, and in total, 5.87 median-length subreads (142x) were used for genome assembly. The average and N50 lengths of these median-length subreads were $16.12 \mathrm{~kb}$ and $26.90 \mathrm{~kb}$, respectively. Illumina sequencing produced $301.05 \mathrm{M}$ paired-end reads $(141 \mathrm{x})$, which were used to estimate genome size and polish the assembly. $382.80 \mathrm{M}$ paired-end reads were obtained from Hi-C sequencing. After discarding duplicated reads and reads without the ligation motif, $165.75 \mathrm{M}$ paired-end reads $(43.30 \%$ ) were used for contig anchoring (SupTab 3 ), and the long-range ( $>20 \mathrm{~kb}$ ) read pairs accounted for $6.02 \%$ based on the final assembly (Tov-G3).

The genome size was estimated by the k-mer based method. The model fit well and explained at least $98.67 \%$ of the sum of squares. The genome size, heterozygosity and unique sequence percentage were estimated to be $639.25 \mathrm{Mb}, 0.13 \%$ and $93.8 \%$, respectively. The primary assembled contigs (Tov-G1) were highly contiguous (SupTab 2 ), with an N50 of $23.14 \mathrm{Mb}$. Additionally, there were no contaminants or mitochondrial sequences detected (SupFig 2 ). The final assembly (Tov-G3) contained 24 pseudochromosomes (Chr1Chr24), including 662.70 Mb (SupTab 4 ). 98.24\% of the bases in the 24 pseudochromosomes had a score above 30 (SupTab 7 ). Completeness was assessed by BUSCO against the database of universal single-copy orthologues in Actinopterygii version10 (Sim O et al. , 2015). A total of $97.8 \%$ of complete genes were de- 
tected, of which $0.8 \%$ were duplicates(SupTab 5 ). The number and total length of gap (filled with N) were 32 and $12.81 \mathrm{~kb}$, respectively. Approximately $43.30 \%$ of reads from Hi-C (Belton et al . 2012) were mapped to the reference genome with a mapQ score of 30 . To construct a genetic linkage map, we produced a full-sib family of 100 offspring (SupFig 3 ). The sex averaged maps were based on 3,307 segregating sites (689,977 SNPs). The total map length was $1984.40 \mathrm{cM}$. There was perfect one-to-one correspondence between the linkage groups and the pseudochromosomes, and a total of 689,070 SNPs from the pseudochromosomes were assigned to the corresponding linkage groups (Figure 1 and SupTab 6 ). The average Pearson correlation coefficient between the SNP orders in pseudochromosome and linkage group pairs was 0.98 . The extremely high ratio of mapped reads to the reference (Tov-G3) also indicates a high quality of the final assembly (SupTab 1 ), especially $99.57 \%$ of the Illumina genome sequencing reads could be mapped back to the genome assembly.

Transposable elements were detected using the homology-based method based on Dfam_3.0 database (Hubley et al . 2016) and amounted to only $4.17 \%$ of the assembly (SupTab 8 ). Retroelements and DNA transposons accounted for $2.00 \%$ and $2.14 \%$, respectively. Simple repeats accounted for $2.75 \%$. To annotate the genes, two rounds of prediction with MAKER software (Cantarel et al. , 2008) were performed, producing 25,720 protein-coding genes (SupTab 9 ). The average gene, coding, exon, and intron lengths were $10669.68 \mathrm{bp}$, $1623.82 \mathrm{bp}, 169.81 \mathrm{bp}$ and $924.85 \mathrm{bp}$, respectively, comparable to those of 14 well-annotated fish species (SupTab 10 ). Approximately $91.2 \%$ of complete BUSCOs were detected among the annotated genes, which is also comparable to the value for above mentioned 14 fish species (SupTab 11). A total of 24,177 genes $(94.00 \%)$ were annotated with putative functions, of which $64.63 \%$ and $67.90 \%$ were assigned with gene ontology and KEGG orthology terms, respectively (SupTab 12 ) (Emms, Kelly, 2019). Non-coding RNA prediction yielded 1,155 tRNAs, 431 rRNAs, 188 snoRNAs, 356 snRNAs, 4 lncRNAs, 961 microRNAs and 407 cis-regulatory elements, with a total length of $369.6 \mathrm{~kb}$ (SupTab 13 ).

\subsection{Divergence time between $T$. ovatus and $S$. dumerili was estimated}

The primary protein sequences of 14 fish species were obtained from Ensemble release 2014 (SupTab 14 ). In each species, there were approximate 20,000 high confidence genes. 349,091 genes (95.0\%) were assigned to 19,804 orthogroups. There were 8,475 orthogroups with representation in all species, and 3,093 of these orthogroups consisted entirely of single-copy genes (SupTab 15-18).

Phylogenetic trees of these 15 species were constructed with all the genes using OrthoFinder2 (Emms, Kelly, 2019) and with 4Dsites (352,840 bp) from single-copy genes using IQ-TREE (Nguyen et al. , 2015) (SupFig 4 ). The two trees were identical, and all the nodes had 100\% bootstrap support. T. ovatus and S. dumerilidiverged 57.45 million years ago $\left(40.82^{\sim} 74.32,95 \%\right.$ confidence interval) based on the independent rates model. The topological structure and divergence times were also supported by other research with common species Danio rerio , Oryzias latipes ,Gasterosteus aculeatus, Takifugu rubripes, and Tetraodon nigroviridis (Yang et al. , 2016).

\subsection{Hsd17b1 was detected as the sex-determining gene with a different sex defining SNP}

1,006 offspring in five full-sib families (Guo et al . 2020) were sampled, of which 536 were females and 547 were males (Table 1 ). Their average weight (mean+-SD) was $934.99+-164.38 \mathrm{~g}$. The sex ratio in each full-sib family was 1:1 ( $p$ value $=0.74$, chi-square test). However, body weight was different between the sexes ( $p$ value $=0.00$, Analysis of Variance) and families ( $p$ value $=0.00$, Analysis of Variance).

Sex linked QTL was detected by QTL-mapping in one full-sib family of 100 progeny using a LOD score of 4.4 and by GWAS analysis in a natural population of 82 individuals using a $p$ value of $10^{-10}$. Only one locus on linage group 11 (Chr16) was consistently and exclusively associated with phenotypic sex (SupFig $\mathbf{5}$ and Figure 2a ). The most significant QTL explained $50.3 \%$ of the phenotype variance, spanned from 38.00 to $66.01 \mathrm{cM}$ on linkage group 11 (Chr16) with a peak at $53.50 \mathrm{cM}$. Multiple QTL mapping confirmed that this interval contained only one QTL. The GWAS analysis detected the same locus and identified a single SNP (Chr16:18219150) that was perfectly and exclusively associated with sex $\left(p\right.$ value $\left.=1.36 \times 10^{-19}\right)$, in which the association has been developed as a sex specific marker and validated in 218 individuals from 
two independent populations previously (Guo et al. , 2021). At this SNP, all females (45 individuals) were heterozygous (G/A) and all males (37 individuals) were homozygous (A/A), suggesting a sex-determining system of female heterogamety.

Coding sequences of $H s d 17 b 1$ from representative vertebrates were collected (SupTab 20 ), including the Wlinked coding sequences in $T$. ovatus, $S$. dumerili and $S$. quinqueradiata, and codon-based test of neutrality between sequences shows that non-synonymous substitution ratio is significantly lower than synonymous substitution ratio $(\mathrm{dN}-\mathrm{dS}<0, \mathrm{p}<0.01$, SupTab 21 ), which indicated these coding sequences were under purifying selection and the allele $\mathrm{G}$ of the sex defining SNP was the ancestral allele and the allele A was the derived allele.

The Z-linked mutations in T. ovatus (Chr16:18219150) and S. dumerili (Chr16:18219853 or SNP1191) (Koyama et al. , 2019) were different and derived, and not detected in four closely related species T. blochii $(\mathrm{n}=15)$, "T. ovatus" $(\mathrm{n}=8$, a species from the Mediterranean which is genetically different from but shares the same scientific name with T. ovatus from South China Sea analyzed in the current study, SupFig 1 ), Caranx ignobilis $(\mathrm{n}=1)$ and C. melampygus $(\mathrm{n}=1)$ in the same family (Figure 3 ).

\subsection{Alternative splicing of Hsd17b1 was confirmed}

Comparing coding sequences of Hsd17b1 in teleosts, the perfectly associated SNP was located in the alternative splice donor site ( $G$ T-AG) of the first intron (Figure 4 ). PacBio Isoform sequencing (Iso-seq) was performed to detect full-length transcripts and RNA-seq was also performed to quantify the transcripts' expression (SupFig 6 and SupTab 19 ). In the full-length non-chimeric sequences (FLNC), two transcripts were obtained from a one-year-old fish's ovary and no transcript was detected from a one-year old male's testis even aligning the raw reads to the genome assembly, which may be caused by Iso-seq's low capacity. RNA-seq confirmed that W-linked transcript was mainly expressed in ovarian tissues and testicular tissues only express the Z-linked transcript (Figure 4a ). Comparing the two transcripts confirmed alternative 5' splice site selection resulted in a intron retention (64 bp) in the Z-linked transcript, shifting the coding frame and introducing a premature termination codon.

\subsection{Sex chromosomes in T. ovatus are undifferentiated}

Comparing depth of coverage and SNP density in resequencing data sets of male and female is an effective way to identify highly diverged region between sex chromosomes in which one member of the pair is moderately or highly degenerated, and has lost many genes (Smeds et al. , 2014). The pseudoautosomal region should show similar coverage in both sexes, and the non-recombination recombining region of $\mathrm{Z}$ chromosome may have degenerated, and might then show a twofold coverage difference. The depth of coverage in the parents from the full-sib family of $T$. ovatus were approximately equal (Figure 2 and SupFig 8c ). And high linkage was not observed around the perfectly associated SNP (SupFig 8a-b ). Also there was no obvious accumulation of simple repeats or other fixed mutations.

\subsection{Knockout of Hsd17b1 in zebrafish leads to "all male"}

To understand the function of $H s d 17 b 1$, two mutant zebrafish lines were established (Figure 5, SupTab 22, Hsd17b1 \#-5bp and Hsd17b1 \#-2+7bp). Both the modified sites were located within the first exon, leading to frameshift mutations. Sex was confirmed through histological examination. We confirmed that all the homozygous $H s d 17 b 1$-deficients were biological males (20 individuals for lineHsd17b1 \#-5bp and 12 individuals for line $H s d 17 b 1$ \#-2+7bp) and heterozygous $H s d 17 b 1$-deficients can develop into female and male (2 males and 3 females for line Hsd17b1 \#-5bp, 2 females and 2 males for line Hsd17b1 \#-2+7bp). Furthermore, there was a slight difference in the fertilization rate between the wild-type and homozygous Hsd17b1 -deficient males ( $p=0.047$, Mann-Whitney test, n [?] 3, SupTab 23 ).

\subsection{Evolution of $\mathrm{Hsd17b1}$ was simulated}

Based on the hypothesis that the W-linked haplotypes of Hsd17b1 was under purifying selection, Z-linked haplotypes may evolved near neutrally and missense mutation, as well as alternative splicing mutation, 
would lead to transformation from W-linked haplotypes to Z-lined haplotypes, we simulated the evolution process of this gene (introns and exons of Hsd17b1 , $1678 \mathrm{bp}$ ) (Table 2 ), At the end of the simulations, recombination suppression has not been established and the the nucleotide diversity of the whole population and the divergence between Z-linked and W-linked Hsd17b1 were positively correlated with the population size (Figure 6.a-f and SupFig9-10 ). The single inactivating mutation dominating the sex determination and the turnover of the dominating sex-determining mutation were observed (Figure 6. g-i and SupFig 11-13).

\section{DISCUSSION}

As shown in this investigation, balanced sex ratio was observed in different families of T. ovatus . QTLmapping and GWAS analyses both identified the same sex QTL. Although lacking supportive evidence of specific expression in undifferentiated gonad and successful sex reversal via knock-in and knock-out methods, the exclusive association of $H s d 17 b 1$ with sex highly supports the sex-determining role of $H s d 17 b 1$ in $T$. ovatus and S. dumerili (Koyamaet al. , 2019). The sex chromosomes of these two species are colinear (SupFig 7 ), however, the sex defining SNPs are different, and the Z-linked alleles are derived and do not present in the closely related species of the same subfamilies, i.e., Trachinotinae and Caranginae (Figure 3 ). It is possible and parsimonious that $H s d 1^{7} \mathrm{~h} b 1$ was the sex-determining gene in the common ancestor of $T$. ovatus and $S$. dumerili, and that sex-determining mutation turnover occurred during evolution. Turnover of sex-determining gene located on different chromosomes in closely related species is common (reviewed by Ponnikas et al . 2018). Turnover on the same chromosome has also been reported in grass puffer (Takifugu niphobles ) (Ieda et al. , 2018). However, turnover of the sex-determining mutation in the same gene has not been previously elaborated. Usually, differential expression of transcripts was tested at the early stage of differentiation to support the role of sex-determining gene. However, the capacity of hybridization in situ to distinguish base difference less than $150 \mathrm{bp}$ (here is $64 \mathrm{bp}$ ) is limited (Lee et al. , 2013) and RNA-seq always tests the expression from the whole trunk instead of the gonad, such as in $S$. dumerili(Koyama et al. , 2019), the tiger pufferfish (Takifugu rubripes) (Kamiya et al. , 2012) and medaka (Oryzias luzonensis ) (Myosho et al. , 2012), which is still indirect evidence. Alternatively, we targeted the gonad to test the alternative splicing. With the facts that $99 \%$ of splice sites are canonical GT-AG pairs (Burset et al. , 2000) and alternative splicing is clearly observed in one-year gonad, these two different isoforms are assumed to result in male and female development, respectively.

In $S$. dumerili and $T$. ovatus, $\mathrm{W}$-linked coding sequences of $H s d 17 b 1$ were conserved across vertebrates. $H s d 1 \% b 1$ functions as a homodimer to catalyze the interconversion between estrogens and androgens (Andersson, 1995) and estrogens in fish are needed not only for ovarian differentiation but also for ovarian maintenance (Guiguen et al. , 2009; Li et al. , 2019). Based on previous research especially in rainbow trout (Baron D et al. , 2004; Bertho et al. , 2018), a positive regulatory loop was proposed to explain the female sex-determining mechanism, in whichfoxl2 induces cyp19a1a expression and thus increases estrogens synthesis that will in return stimulates the expression offoxl2. Synthesis of estradiol from androstenedione in vivo follows $\Delta 4$ (Cyp19a1a and Hsd17b1) or $\Delta 5$ (Hsd17b3and Cyp19a1a) pathways that have been reported in several fish (Tenugu et al. , 2021). Previous researches show that knockout of Cyp19a1a leads to "all male" phenotype in zebrafish and female-to-male reverse in XX tilapia (Li et al. , 2013; Zhanget al. , 2017). Similarly, "all male" phenotype of zebrafish was produced in this study following the successful knockout of Hsd17b1. Based on the consistent consequence of male development with the spontaneous mutants in $S$. dumerili and $T$. ovatusand artificial mutation in zebrafish, the $\Delta 4$ pathway is considered crucial during early sex differentiation and this mechanism is conserved in teleosts. Together, these results show that conserved $H s d 17 b 1$ was essential for the female development in teleosts.

Dominant negative of a sex-determining gene was described in African clawed frogs (Xenopus laevis ) with a ZZ/ZW sex determination system, where Dmrt1 is conserved in male development, while its duplicate, DM$W$, is W-linked and a dominant-negative regulator ofDmrt1 . Although they share similar DNA-binding properties, $D M-W$ loses the transactivating domain and dominantly represses male development (Yoshimoto, Ito, 2011). In S. dumerili, the Z-linked missense mutation of SNP1196 caused a change from glycine (G144) 
to glutamic acid (E144) in Z-linked $H S D 17 B 1$ protein and led to $99.66 \%$ reduction of catalytic activity based on molecular dynamics simulations and steroid conversion assay (Koyama et al. , 2019). In addition, SNP1195 was linked to Z-allele and resulted in the transition of G114 to lysine (K144) at a low frequency $(\sim 1 \%)$, which was also assumed to result in malfunction based on molecular dynamics simulations (Koyama et al. , 2019). InT. ovatus, Z-linked allele in the sex defining SNP (Chr16:18219150) was assumed to result in the intron retention $(64 \mathrm{bp})$, the reading frame shrift and the introduction of a premature termination codon. In addition, a Z-linked non-synonymous mutation (Chr16:18220639, from P to H) located in the sixth exon was also observed in a male individual of the natural population. Notably, the sex defining SNPs in $S$. dumerili and $T$. ovatus are different, which should be mutually exclusive if Z-linked haplotype functions as dominate negative. Combining the phenotype "all male" in homozygous mutants ofHsd17b1 in zebrafish, it is likely that $H s d 17 b 1$ is not necessary for male development and the Z-linked haplotypes in $S$. dumerili and $T$. ovatus may evolve near neutrally.

Sex-determining genes must be subject to negative selection, which was left out in the neutral model (Jeffries et al. , 2021). As described above, W-linked haplotypes of Hsd17b1 is assumed to be under purifying selection, while Z-linked haplotypes may evolve near neutrally. This suggests that conserved W-linked haplotypes could transform to Z-linked haplotypes caused by loss-of-function mutation. The local recombination rate between $\mathrm{W}$ and $\mathrm{Z}$ chromosomes is estimated to be $2.15 \times 10^{-6}$ in female T. ovatus (Figure $\mathbf{2 b}$ and SupFig $\mathbf{8 b}$ ), two order of magnitudes higher than the general mutation rate of $1.61 \times 10^{-8}$ (Lipson et al. , 2015). In the simulation analysis of $H s d 17 b 1$ evolution, turnovers of sex determining mutation and single sex defining mutation dominating sex determination were observed (Figure 6g-i, SupFig11-13), which is consistent to the observations in T. ovatus and $S$. dumerili, in return, supports our hypothesis. Specifically, the relatively low level of genetic diversity (0.13\%) of T. ovatus(SupFig 14 and SupTab 24 ) means a relatively strong force of drift to remove other minor malfunctional mutations when the genetic diversity was from $0.10 \%$ to $0.15 \%$ according to the simulations (Figure $\mathbf{5} \mathbf{b}$ and $\mathbf{h}$ ), which is supported by approximate $100 \%$ accurate rate using this site (Chr16:18219150) as a sex specific marker (Guo et al. , 2021).

The sex chromosomes are homologous and undifferentiated except the sex defining SNP in S. dumerili(Koyama et al. , 2019) and T. ovatus, it is possible and parsimonious that the state of undifferentiation persists for 57 million years from the common ancestor. This situation is different from the convergence of recombination loss and does not totally fit those models, i.e. high turnover of sex chromosome, Fountainof-Youth, and the neutral model. In the neutral model, when the mutation rate $\left(1 \times 10^{-6}\right)$ was equal to the recombination rate $\left(1 \times 10^{-6}\right)$, sex chromosome would maintain undifferentiated (Jeffries et al. , 2021). In our simulations, relatively high recombination rate compared to mutation rate would contribute to lower divergence between W-linked and Z-linked haplotypes(SupFig 9 and 10) and the divergence was positively correlated with the population size (Figure 6d-f ). which hints that transformations from conserved Wlinked haplotypes to Z-linked haplotypes and relatively strong forces of drift and recombination contribute to maintaining undifferentiation of sex chromosomes in micro-evolutionary scale. However, only point mutations were taken into consideration and insertion or deletion always occurs, which has stronger effects in suppressing recombination. It is supposed that a 241-bp deletion in turquoise killifish (Nothobranchius furzeri ) (Reichwald et al. 2015) and a large inversion in nine-spined stickleback (Pungitius pungitius) (Natri et al. 2019) induce recombination suppression and lead to establishment of non-recombination region. Seen from the sequence alignment of Hsd17b1 between T. ovatusand S. dumerili (SupFig 15), length difference has been accumulated in the first and firth intron, which shows that insertions or deletions occurred in introns have been swept or fixed within species of $T$. ovatus and $S$. dumerili while those occurred in coding sequences have been swept due to drift. Furthermore, our hypothesis may also apply to the tiger pufferfish, in which homozygous form of less-effective and derived allele (C/C) in sex-determining geneAmhr2 (site 7271) leads to female development while heterozygous form of the alleles $(\mathrm{C} / \mathrm{G}$ ) leads to male development (Kamiya et al. 2012). The mechanism may be that purifying selection on Y-lined haplotypes, transformations from conserved Y-linked haplotypes to X-linked haplotypes and neutral forces contribute to the undifferentiation of sex chromosomes, although the degree of deviation from neutrality of $\mathrm{X}$-linked haplotype is not clear.

Although simulation analysis supports our hypothesis that selection, transformation and neutral forces con- 
tribute to the undifferentiation of sex chromosomes in micro-evolutionary scale, it is not impossible that the sex-determining gene in the ancestor of the family Carangidae was another gene and convergent turnovers of sex-determining gene occurred recently in $T$. ovatus and $S$. dumerili in macro-evolutionary scale. Convergent turnovers have been observed in true frogs (Jeffries et al. , 2018). The sex determining gene in New Zealand trevally (Pseudocaranx georgianus ), belonging to the subfamily Caranginae and the clade (Naucratinae, Caranginae), is assumed to be a Cyp19b or Cyp19a1a related gene instead ofHsd17b1 (Ruigrok et al. 2021). Nonetheless, it cannot rule out the role of selection, transformation and neutral forces in micro-evolutionary scale in maintaining undifferentiated sex chromosomes.

\section{CONCLUSIONS}

We traced the origin of sex-determining gene of $S$. dumerili in the family Carangidae and showed that $H s d 17 b 1$ gene is the sex determining gene for both $T$. ovatus and $S$. dumerili. The alternative splicing in master sex determining gene was first time reported. The undifferentiated sex chromosome in T. ovatus and $S$. dumerili except their respectively sex defining SNPs parsimoniously suggests a persistence of the undifferentiation for 57 million years. The observation and simulation analysis support that recurrent directional transformations from conserved W-linked haplotypes of the sex-determining gene to Z-linked haplotypes, relatively strong forces of drift and recombination comprehensively contribute to the turnover of primary sex defining mutation and undifferentiation of sex chromosomes.

\section{FUNDING}

This study was supported by the Guangdong Basic and Applied Basic Research Foundation (2019A1515110309), Natural Science Foundation of Hainan Province (320QN362), National Natural Science Foundation of China (U20A2064), the National Key Research and Development Program of China (2018YFD0900303), the Financial Fund of the Ministry of Agriculture and Rural Affairs of China (NHYYSWZZZYKZX2020), the China Agriculture Research System (CARS-48), the China-ASEAN Maritime Cooperation Fund (00-201620821), the Central Public-interest Scientific Institution Basal Research Fund (2020GH06, 2020TD29), and China Agriculture Research System of MOF and MARA (CARS-47).

\section{AVAILABLITY OF DATA}

The genome assembly: JACGTO000000000. Genome sequencing: SRR12380975 (PacBio genome sequencing), SRR12380974 (Illumina genome sequencing), SRR12380973 (PacBio transcriptome sequencing), and SRR8168440 (Hi-C sequencing). Iso-seq: SRR14553106 (female), SRR14553104 (male). RNA-seq: SRR6168954 (female), SRR14553107(female), SRR14553103(female), SRR6168955 (male), SRR14553105 (male), SRR14553102 (male). Resequencing: PRJNA552381 (full-sib family), PRJNA730209 (natural population, including T. ovatus $(\mathrm{n}=82$, South China Sea), T. blochii $(\mathrm{n}=15)$ and T. ovatus $(\mathrm{n}=8$, Mediterranean)), SRR1002927 (Caranx ignobilis ), SRR1002928 (Caranx melampygus ). W-linked Hsd17b1 coding sequence: OK170040, Z-linkedHsd17b1 coding sequencing: OK170041.

\section{AVAILABILITY OF CODE}

The code for simulation of turnover of sex-determining mutation are available at https://github.com/zsdxgl/Turnover-of-sex-mutation.

\section{AUTHOR CONTRIBUTIONS}

Guo analyzed the data and wrote the manuscript. D. Malara and P. Battaglia collected the samples of T. ovatus (Mediterranean). L. Guo, J. Yang, N. Zhang, BS. Liu, K. Zhu, H. Guo, B. Liu and F. Zhao collected the sequencing samples (South China Sea), extracted the DNA/RNA and performed genome sequencing. All the authors contributed to manuscript revision.

\section{SUPPLEMENTARY MATERIAL}

Supplementary material is available online.

\section{COMPETING INTERESTS}


No competing interests.

\section{References}

Altschul SF, Madden TL, Schaffer AA, et al. (1997) Gapped BLAST and PSI-BLAST: a new generation of protein database search programs.Nucleic Acids Res 25 , 3389-3402.

Andersson S (1995) 17 beta-hydroxysteroid dehydrogenase: isozymes and mutations. Journal of endocrinology $146,197-200$.

Apweiler R, Bairoch A, Wu CH, et al. (2004) UniProt: the universal protein knowledgebase. Nucleic acids research 32 , D115-D119.

Azzi A, Rehse PH, Zhu DW, et al. (1996) Crystal structure of human estrogenic 17 beta-hydroxysteroid dehydrogenase complexed with 17 beta-estradiol. Nature structural biology $\mathbf{3}, 665$.

Bao L, Tian C, Liu S, et al. (2019) The Y chromosome sequence of the channel catfish suggests novel sex determination mechanisms in teleost fish. BMC Biology 17,6.

Baron D, Cocquet J, Xia X, et al. (2004) An evolutionary and functional analysis of FoxL2 in rainbow trout gonad differentiation. J Mol Endocrinol 33, 705-715.

Benson G (1999) Tandem repeats finder: a program to analyze DNA sequences. Nucleic acids research $\mathbf{2 7}$, 573.

Benton MJ, Donoghue PC (2007) Paleontological evidence to date the tree of life. Mol Biol Evol 24, 26-53.

Bertho S, Herpin A, Branthonne A, et al. (2018) The unusual rainbow trout sex determination gene hijacked the canonical vertebrate gonadal differentiation pathway. Proceedings of the National Academy of Sciences $115,12781-12786$.

Burset M, Seledtsov IA, Solovyev VV (2000) Analysis of canonical and non-canonical splice sites in mammalian genomes. Nucleic acids research $\mathbf{2 8}, 4364-4375$.

Cantarel BL, Korf I, Robb SMC, et al. (2008) MAKER: an easy-to-use annotation pipeline designed for emerging model organism genomes. Genome research $18,188-196$.

Capel B (2017) Vertebrate sex determination: evolutionary plasticity of a fundamental switch. Nat Rev Genet 18, 675-689.

Chen S, Zhang G, Shao C, et al. (2014) Whole-genome sequence of a flatfish provides insights into ZW sex chromosome evolution and adaptation to a benthic lifestyle. Nature Genetics 46 , 253-260.

Chin CS, Alexander DH, Marks P, et al. (2013) Nonhybrid, finished microbial genome assemblies from long-read SMRT sequencing data.Nat Methods 10, 563-569.

Chin CS, Peluso P, Sedlazeck FJ, et al. (2016) Phased diploid genome assembly with single-molecule realtime sequencing. Nat Methods13, 1050-1054.

Damerau M, Freese M, Hanel R (2018) Multi-gene phylogeny of jacks and pompanos (Carangidae), including placement of monotypic vadigo Campogramma glaycos. J Fish Biol 92 , 190-202.

Danecek P, Auton A, Abecasis G, et al. (2011) The variant call format and VCFtools. Bioinformatics 27 , 2156-2158.

Devlin RH, Nagahama Y (2002) Sex determination and sex differentiation in fish: an overview of genetic, physiological, and environmental influences, pp. 191-364. Elsevier B.V, Amsterdam.

Dong S, He W, Ji J, et al. (2021) LDBlockShow: a fast and convenient tool for visualizing linkage disequilibrium and haplotype blocks based on variant call format files. Briefings in Bioinformatics 22 . 
Dos Reis M, Yang Z (2019) Bayesian molecular clock dating using genome-scale datasets. Methods in molecular biology (Clifton, N.J.)1910, 309-330.

Durand NC, Shamim MS, Machol I, et al. (2016) Juicer provides a one-click system for analyzing loopresolution Hi-C experiments. Cell Systems 3 , 95-98.

Emms D, Kelly SL (2019) OrthoFinder: phylogenetic orthology inference for comparative genomics. Genome Biology $20,238$.

English AC, Richards S, Han Y, et al. (2012) Mind the gap: upgrading genomes with Pacific Biosciences RS long-read sequencing technology.PloS one 7 , e47768.

Flicek P, Amode MR, Barrell D, et al. (2013) Ensembl 2014. Nucleic acids research 42, D749-D755.

Frazee AC, Pertea G, Jaffe AE, et al. (2015) Ballgown bridges the gap between transcriptome assembly and expression analysis. Nat Biotechnol 33, 243-246.

Froese R, Pauly D (2011) FishBase.World Wide Web electronic publication.www.fishbase.org, version.(accessed on 3 Sup 2019).

Gasteiger E, Gattiker A, Hoogland C, et al. (2003) ExPASy: the proteomics server for in-depth protein knowledge and analysis. Nucleic acids research 31, 3784-3788.

Giles S, Xu G, Near TJ, Friedman M (2017) Early members of 'living fossil' lineage imply later origin of modern ray-finned fishes. Nature549, 265-268.

Gonzalez-Garay ML (2015) Introduction to isoform sequencing using Pacific Biosciences Technology (IsoSeq), pp. 141-160. Springer Netherlands, Dordrecht.

Graves (2015) In retrospect: Twenty-five years of the sex-determining gene.Nature 528, 343-344.

Grossen C, Neuenschwander S, Perrin N (2012) The evolution of XY recombination: sexually antagonistic selection versus deleterious mutation load.Evolution 66 , 3155-3166.

Guiguen Y, Bertho S, Herpin A, Fostier A (2018) Sex determination and sex control in Salmonidae. In: Sex Control in Aquaculture (eds. Wang HP, Piferrer F, Chen SL, Shen ZG), pp. 249-280.

Guiguen Y, Fostier A, Piferrer F, Chang C (2009) Ovarian aromatase and estrogens: A pivotal role for gonadal sex differentiation and sex change in fish. General and comparative endocrinology 165, 352-366.

Guo L, Xu Y, Zhang N, et al. (2019) A high-density genetic linkage map and QTL mapping for Sex in Black Tiger Shrimp (Penaeus monodon ).Frontiers in Genetics $10,326$.

Guo L, Yang J, Liu B, et al. (2021) Colinearity based sex-specific marker development in the golden pompano (Trachinotus ovatus).Aquaculture $\mathbf{5 4 4}, 737044$.

Guo L, Yang Q, Yang JW, et al. (2020) MultiplexSSR: A pipeline for developing multiplex SSR-PCR assays from resequencing data.Ecology and Evolution 10 , 3055-3067.

Hu S, He M, Zhang H, Wang Y, Liufu Y (2007) Study on the karyotyre in the Trachinotus ovatus . Journal of Guangzhou University (Natural Science Edition) 6 , 23-25.

Hubley R, Finn RD, Clements J, et al. (2016) The Dfam database of repetitive DNA families. Nucleic Acids Res 44 , D81-D89.

Huerta-Cepas J, Forslund K, Coelho LP, et al. (2017) Fast genome-wide functional annotation through orthology assignment by eggNOG-Mapper.Molecular Biology and Evolution 34, 2115-2122.

Ieda R, Hosoya S, Tajima S, et al. (2018) Identification of the sex-determining locus in grass puffer (Takifugu niphobles ) provides evidence for sex-chromosome turnover in a subset of Takifugu species. PLOS ONE 13 , e190635. 
Jeffries DL, Gerchen JF, Scharmann M, Pannell JR (2021) A neutral model for the loss of recombination on sex chromosomes. Philosophical Transactions of the Royal Society B: Biological Sciences 376 , 20200096.

Jeffries DL, Lavanchy G, Sermier R, et al. (2018) A rapid rate of sex-chromosome turnover and non-random transitions in true frogs. Nature Communications $\mathbf{9}$.

Jiang X, Wei P, Chen X, et al. (2015) Histological observation of Trachinotus ovatus and methodical construction of simple method of early sex identification. Southwest China Journal of Agricultural Sciences $\mathbf{2 8}$ , 428-432.

Kalvari I, Nawrocki EP, Argasinska J, et al. (2018) Non-coding RNA analysis using the Rfam database. Current protocols in bioinformatics $\mathbf{6 2}$, e51.

Kamiya T, Kai W, Tasumi S, et al. (2012) A trans-species missense SNP in Amhr2 is associated with sex determination in the tiger pufferfish, Takifugu rubripes (Fugu). PLoS Genetics 8, e1002798.

Keibler E, Brent MR (2003) Eval: a software package for analysis of genome annotations. BMC Bioinformatics 4,50 .

Kikuchi K, Hamaguchi S (2013) Novel sex-determining genes in fish and sex chromosome evolution. Developmental Dynamics 242 , 339-353.

Kim D, Langmead B, Salzberg SL (2015) HISAT: a fast spliced aligner with low memory requirements. Nat Methods $12,357-360$.

Kim D, Paggi JM, Park C, Bennett C, Salzberg SL (2019) Graph-based genome alignment and genotyping with HISAT2 and HISAT-genotype. Nat Biotechnol 37, 907-915.

Kolbe DL, Eddy SR (2009) Local RNA structure alignment with incomplete sequence. Bioinformatics 25 , 1236-1243.

Koopman P, Gubbay J, Vivian N, Goodfellow P, Lovell-Badge R (1991) Male development of chromosomally female mice transgenic for Sry.Nature 351, 117-121.

Korf I (2004) Gene finding in novel genomes. BMC Bioinformatics5 , 59.

Kovaka S, Zimin AV, Pertea GM, et al. (2019) Transcriptome assembly from long-read RNA-seq alignments with StringTie2. Genome Biol20 , 278.

Koyama T, Nakamoto M, Morishima K, et al. (2019) A SNP in a steroidogenic enzyme is associated with phenotypic sex in Seriola fishes. Current Biology 29 , 1901-1909.

Kuraku S, Zmasek CM, Nishimura O, Katoh K (2013) aLeaves facilitates on-demand exploration of metazoan gene family trees on MAFFT sequence alignment server with enhanced interactivity. Nucleic acids research41, W22-W28.

Lee D, Xiong S, Xiong W (2013) General introduction to In Situ hybridization protocol using nonradioactively labeled probes to detect mRNAs on tissue sections, pp. 165-174. Humana Press, Totowa, NJ.

Li H (2016) Minimap and miniasm: fast mapping and de novo assembly for noisy long sequences. Bioinformatics 32, 2103-2110.

Li H (2018) Minimap2: pairwise alignment for nucleotide sequences.Bioinformatics 34 , 3094-3100.

Li H, Handsaker B, Wysoker A, et al. (2009) The sequence alignment/map format and SAMtools. BIOINFORMATICS 25, 2078-2079.

Li M, Sun L, Wang D (2019) Roles of estrogens in fish sexual plasticity and sex differentiation. General and Comparative Endocrinology277, 9-16. 
Li M, Yang H, Li M, et al. (2013) Antagonistic roles of Dmrt1 and Foxl2 in sex differentiation via estrogen production in tilapia as demonstrated by TALENs. Endocrinology (Philadelphia)154, 4814-4825.

Lipson M, Loh PR, Sankararaman S, et al. (2015) Calibrating the human mutation rate via ancestral recombination density in diploid genomes.PLoS Genet $\mathbf{1 1}$, e1005550.

Mank JE, Avise JC (2009) Evolutionary diversity and turn-over of sex determination in teleost fishes. Sex Dev $3,60-67$.

Marcais G, Kingsford C (2011) A fast, lock-free approach for efficient parallel counting of occurrences of k-mers. Bioinformatics 27 , 764-770.

Matthews BJ, Dudchenko O, Kingan SB, et al. (2018) Improved reference genome of Aedes aegypti informs arbovirus vector control.Nature $\mathbf{5 6 3}, 501-507$.

Mei J, Gui J (2015) Genetic basis and biotechnological manipulation of sexual dimorphism and sex determination in fish. Science China Life Sciences 58 , 124-136.

Mei W YGLJ (2018) basicTrendline: Add trendline and confidence interval of basic regression Models to Plot.

Miura I (2018) Sex determination and sex chromosomes in amphibia. Sexual development 11, 298-306.

Modi WS, Crews D (2005) Sex chromosomes and sex determination in reptiles: commentary. Current Opinion in Genetics \& Development15, 660-665.

Myosho T, Otake H, Masuyama H, et al. (2012) Tracing the emergence of a novel sex-determining gene in medaka, Oryzias luzonensis .Genetics 191, 163-170.

Myosho T, Takehana Y, Hamaguchi S, Sakaizumi M (2015) Turnover of sex chromosomes in celebensis group medaka fishes. G3 (Bethesda)5 , 2685-2691.

Nei M, Li WH (1979) Mathematical model for studying genetic variation in terms of restriction endonucleases. Proceedings of the National Academy of Sciences - PNAS $\mathbf{7 6}$, 5269-5273.

Nguyen LT, Schmidt HA, von Haeseler A, Minh BQ (2015) IQ-TREE: a fast and effective stochastic algorithm for estimating maximum-likelihood phylogenies. Mol Biol Evol 32, 268-274.

Ooijen JWV, Ooijen J, Ooijen JW (2009) MapQTL 6: Software for the mapping of quantitative trait loci in experimental populations of diploid species.

Pan Q, Feron R, Jouanno E, et al. (2021) The rise and fall of the ancient northern pike master sexdetermining gene. eLife $10,1-30$.

Perrin N (2009) Sex Reversal: A fountain of youth for sex chromosomes? Evolution 63, 3043-3049.

Pertea M, Kim D, Pertea GM, Leek JT, Salzberg SL (2016) Transcript-level expression analysis of RNA-seq experiments with HISAT, StringTie and Ballgown. Nat Protoc 11, 1650-1667.

Ponnikas S, Sigeman H, Abbott JK, Hansson B (2018) Why do sex chromosomes stop recombining? Trends in genetics $\mathbf{3 4}, 492-503$.

Purcell S, Neale B, Todd-Brown K, et al. (2007) PLINK: a tool set for whole-genome association and population-based linkage analyses. The American Journal of Human Genetics 81 , 559-575.

Puttick MN (2019) MCMCtreeR: functions to prepare MCMCtree analyses and visualize posterior ages on trees. Bioinformatics 35 , 5321-5322.

Rastas P (2017) Lep-MAP3: robust linkage mapping even for low-coverage whole genome sequencing data. Bioinformatics 33 , 3726-3732. 
Rezvoy C, Charif D, Gueguen L, Marais GA (2007) MareyMap: an R-based tool with graphical interface for estimating recombination rates.Bioinformatics $23,2188-2189$.

Robert X, Gouet P (2014) Deciphering key features in protein structures with the new ENDscript server. Nucleic acids research $\mathbf{4 2}$, W320-W324.

Rodrigues N, Studer T, Dufresnes C, Perrin N (2018) Sex-chromosome recombination in common frogs brings water to the Fountain-of-Youth. Mol Biol Evol 35 , 942-948.

Romano S, Kaufman OH, Marlow FL (2020) Loss of dmrt1 restores zebrafish female fates in the absence of cyp19a1a but not rbpms2a/b. Development147, v190942.

Ruigrok M, Catanach A, Bowatte D, et al. (2021) The genome of New Zealand trevally (Carangidae: Pseudocaranx georgianus ) uncovers a XY sex determination locus. bioRxiv , 2021-2024.

Santini F, Carnevale G (2015) First multilocus and densely sampled timetree of trevallies, pompanos and allies (Carangoidei, Percomorpha) suggests a Cretaceous origin and Eocene radiation of a major clade of piscivores. Mol Phylogenet Evol 83, 33-39.

Schartl M (2004) Sex chromosome evolution in non-mammalian vertebrates. Current opinion in genetics $\mathcal{E}$ development 14,634-641.

Sim O FA, Waterhouse RM, Panagiotis I, Kriventseva EV, Zdobnov EM (2015) BUSCO: assessing genome assembly and annotation completeness with single-copy orthologs. Bioinformatics 31, 3210-3212.

Smeds L, Kawakami T, Burri R, et al. (2014) Genomic identification and characterization of the pseudoautosomal region in highly differentiated avian sex chromosomes. Nat Commun 5, 5448.

Smith-Vaniz WF, Walsh SJ (2019) Indo-West Pacific species of Trachinotus with spots on their sides as adults, with description of a new species endemic to the Marquesas Islands (Teleostei: Carangidae). Zootaxa4651 , 1-37.

Stanke M, Keller O, Gunduz I, et al. (2006) AUGUSTUS: ab initio prediction of alternative transcripts. Nucleic Acids Research34, W435-W439.

Suyama M, Torrents D, Bork P (2006) PAL2NAL: robust conversion of protein sequence alignments into the corresponding codon alignments.Nucleic Acids Research 34, 609-612.

Tao W, Conte MA, Wang D, Kocher TD (2021) Network architecture and sex chromosome turnovers. BioEssays 43, 2000161.

Tenugu S, Pranoty A, Mamta S, Senthilkumaran B (2021) Development and organisation of gonadal steroidogenesis in bony fishes - A review.Aquaculture and Fisheries 6 , 223-246.

Thomas GWC, Hahn MW (2019) Referee: reference assembly quality scores.Genome biology and evolution $11,1483-1486$.

Thorvaldsdottir H, Robinson JT, Mesirov JP (2013) Integrative Genomics Viewer (IGV): high-performance genomics data visualization and exploration.Briefings in bioinformatics 14, 178-192.

Volff J (2002) Sex determination in fish. Genome Biology 3, s52.

Volff JN, Nanda I, Schmid M, Schartl M (2007) Governing sex determination in fish: regulatory putsches and ephemeral dictators. Sexual development 1 , 85-99.

Vurture GW, Sedlazeck FJ, Nattestad M, et al. (2017) GenomeScope: fast reference-free genome profiling from short reads. Bioinformatics33, 2202-2204.

Walker BJ, Abeel T, Shea T, et al. (2014) Pilon: an integrated tool for comprehensive microbial variant detection and genome assembly improvement. PloS one $\mathbf{9}$, e112963. 
Waterhouse A, Bertoni M, Bienert S, et al. (2018) SWISS-MODEL: homology modelling of protein structures and complexes. Nucleic Acids Res46, W296-W303.

Wright AE, Dean R, Zimmer F, Mank JE (2016) How to make a sex chromosome.Nature communications 7 , 12087 .

Wu TD, Watanabe CK (2005) GMAP: a genomic mapping and alignment program for mRNA and EST sequences. Bioinformatics (Oxford, England)21, 1859-1875.

Yang J, Chen X, Bai J, et al. (2016) The Sinocyclocheilus cavefish genome provides insights into cave adaptation. BMC Biol14, 1.

Yang Z (2007) PAML 4: Phylogenetic analysis by maximum likelihood.Molecular Biology and Evolution 24 , 1586-1591.

Yoshimoto S, Ito M (2011) A ZZ/ZW-type sex determination in Xenopus laevis. The FEBS journal 278 , $1020-1026$.

Zhang D, Guo L, Guo H, et al. (2019) Chromosome-level genome assembly of golden pompano (Trachinotus ovatus ) in the family Carangidae.Scientific Data 6 , 1-11.

Zhang X, Li M, Ma H, et al. (2017) Mutation of foxl2 or cyp19a1a results in female to male sex reversal in XX nile tilapia.Endocrinology (Philadelphia) 158, 2634-2647.

\section{Tables}

Table 1 . The statistics of sex records in five full-sib families.

Table 2 . The parameters and results of the simulation analysis of $H s d 17 b 1$ evolution.

Figures

Figure 1. The chromosome-level genome assembly of T. ovatus . a . The k-mer profile. b . The genetic linkage map constructed with a full-sib family. c . The heatmap of long-range interactions of the chromosomes captured by Hi-C sequencing. The assessment is based on the final assembly (Tov-G3). d . Comparison of genome assembly and genetic linage maps.

Figure 2. The statistics of the sex chromosome. a . The Manhattan plot of the genome-wide association study for the sex. The red point indicates the perfect associated SNP (Chr16:18219150). b . The recombine ratio of the female parent in the mapping family.c . The nucleotide diversity (non-overlapped window, window size $=10 \mathrm{~kb}$ ) in the natural population. $\mathbf{d}$. The comparison of the depth of the coverage (nonoverlapped window, window size $=10 \mathrm{~kb}$ ) in the parents of the full-sib family. e . The non-overlapped scan of the simple repeat sequence (non-overlapped window, window size $=10 \mathrm{~kb}$ ).

Figure 3 . Turnover of sex defining SNPs. a. The positions of the two sex-defining SNPs, Chr16:18219150 in T. ovatus and SNP1196 in $S$. dumerili . b. The turnovers of sex defining SNPs. T. ovatus (South China Sea) and "T. ovatus"(Mediterranean) are different species according to Smith-Vaniz et al .(2019) (SupFig 1 ). We use T. ovatus customarily in this study and distinguish them with the locations. The phylogenetic tree was adopted from Damerau et al . (2018), Santini et al . (2015) and Smith-Vaniz et al .(2019).

Figure 4. The alternative splicing of the gene Hsd17b1 in T. ovatus . a . The schematic diagram of the geneHsd17b1 and Sashimi plot of their expression in ovaries and testes $(\mathrm{n}=3)$. The $\mathrm{W}$-linked transcript was highly expressed in ovaries and the Z-linked transcript, including extra $64 \mathrm{bp}$ at the end of the first exon, was mainly expressed in testis. $\mathbf{b}$. The multiple sequence alignments. The red and the black arrows indicate sex defining mutations in T. ovatus and in S. dumerili, respectively. In T. ovatus, the W-linked protein sequence of Hsd17b1 is conserved across vertebrate, including human and coelacanth, while the intron retention of extra $64 \mathrm{bp}$ in the Z-linked transcript leads to shift of coding frame and introduces a premature terminal codon. The sex defining mutation in Z-linked protein of $S$. dumerili breaks $\eta \mathrm{B}$ helix, and significantly impairs the conversion activity from oestrone to estradiol and finally leads to binary sex development (Koyamaet 
al. , 2019). c . The three dimensional models of human protein crystal structure (ID: 1iol.1), predicted W-linked protein (290 aa) and predicted Z-linked protein (87 aa). The cofactor NADPH (red sticks) and the catalysate estradiol (blue sticks) are also included in the human crystal protein.

Figure 5. Establishment of the Hsd17b1 mutant lines in zebrafish. a . The strategy for establishment of mutation lines.b. The diagram of the mutant lines. c . Histological confirmation of the sex in homozygous mutant lines $(\mathrm{n}=6$, Bar $=50 \mathrm{um})$. All samples were collected at 145 day post fertilization. $\mathbf{d}$. The spawning ratio of wild males and mutant males crossed with wild-type females (n [?] 3).

Figure 6 . Simulation of sex defining mutation turnover. The simulation was initiated with the Wlinked haplotype and Z-linked haplotype of $H s d 17 b 1$ gene region (1678 bp, including exons and introns) and performed with changing from $\mathrm{W}$-linked haplotype to Z-linked haplotype because of missense mutations in coding regions and mutations on alternative splicing sites. With the constraints on computer time, the large population size $(\mathrm{N}, \mathrm{N} \times 100$ and $\mathrm{N} \times 10,000$ in $\mathbf{a}, \mathbf{b}$ and $\mathbf{c}$, respectively) was simulated by rescaling 1 , 100 and 10,000 times of the mutation rate and recombination rate. Divergence $\left(\mathrm{d}_{\mathrm{xy}}\right)$ between the W-lined and Z-linked haplotypes would enlarge along with the population size increasing (e-f ). The turnovers of sex-determining mutation and single mutation dominating sex determination were observed with different sites represented by respective colors ( $\mathbf{g}-\mathbf{i}$ ). Showing in the figures g-i are one of the ten simulations with the population size 200 and three sets of recombination rates $\left(\mathrm{r}_{0}\right)$ and mutation rates $(\mu)$.

\section{Hosted file}

Table 1.docx available at https://authorea.com/users/446431/articles/545673-turnover-of-sexdefining-mutation-provides-an-insight-into-evolution-of-sex-chromosomes-in-the-goldenpompano-trachinotus-ovatus

\section{Hosted file}

Table 2.docx available at https://authorea.com/users/446431/articles/545673-turnover-of-sexdefining-mutation-provides-an-insight-into-evolution-of-sex-chromosomes-in-the-goldenpompano-trachinotus-ovatus
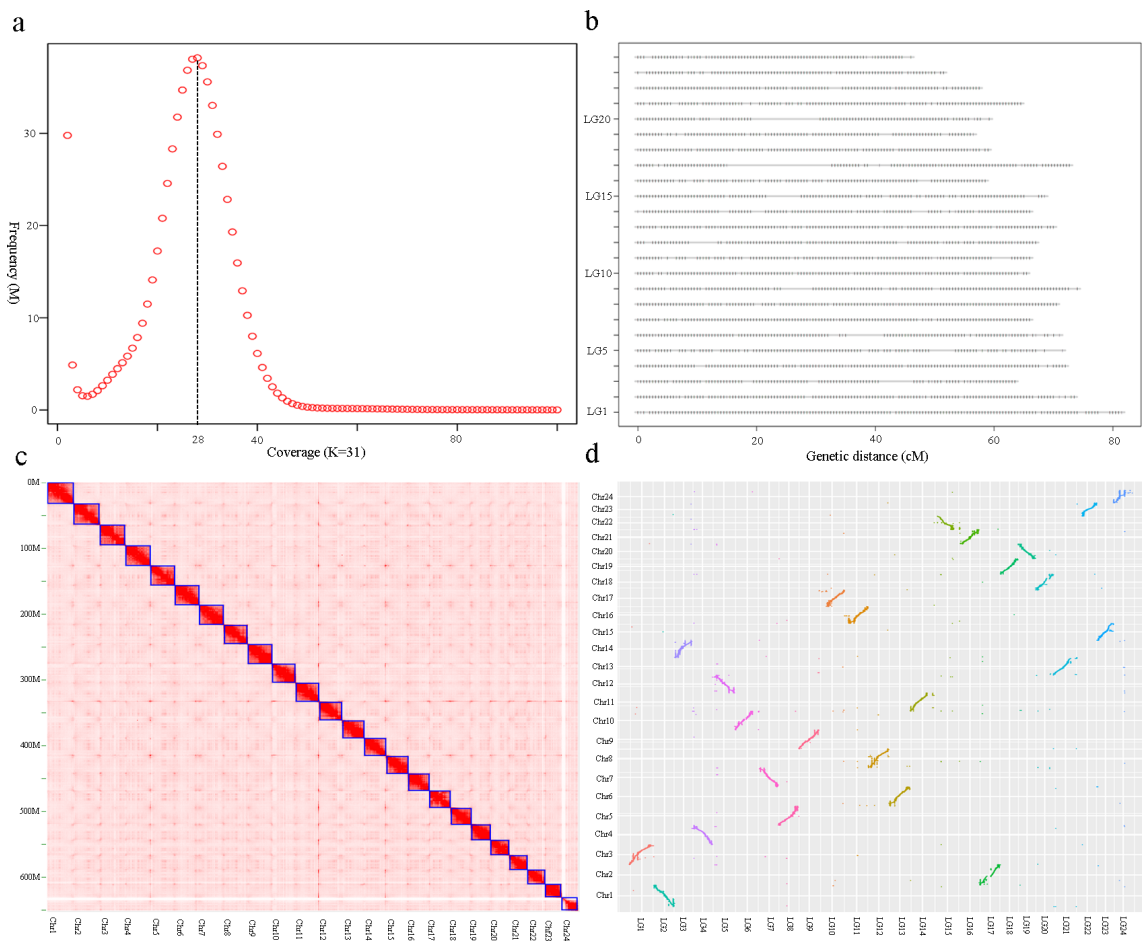


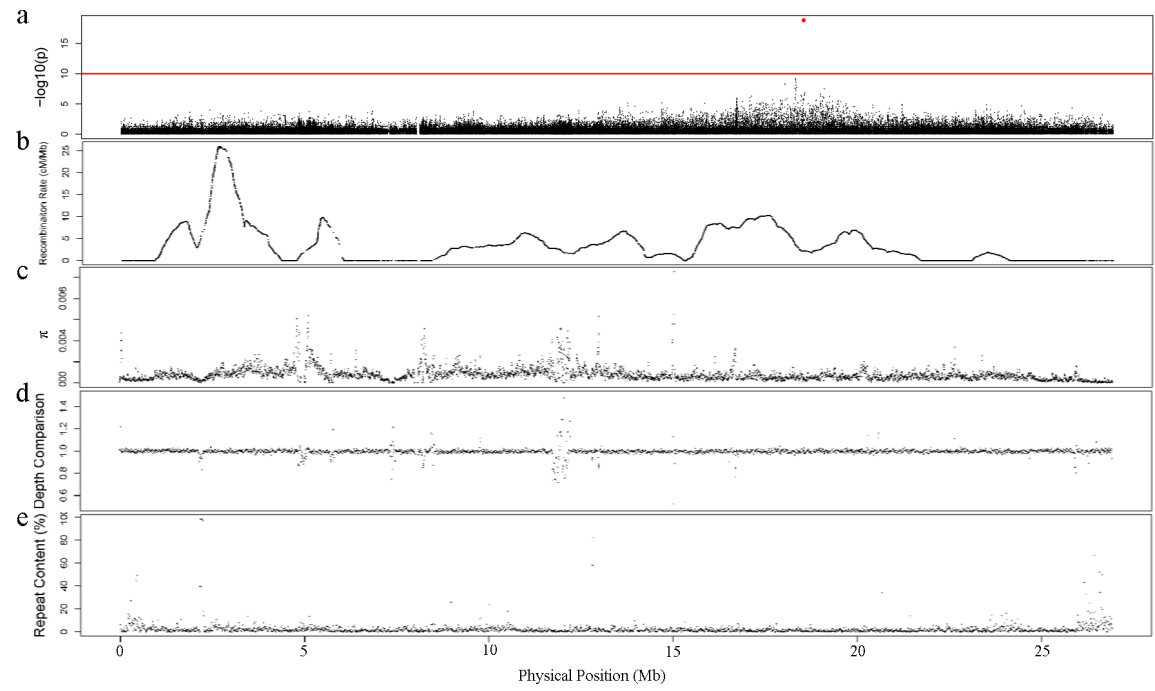

a

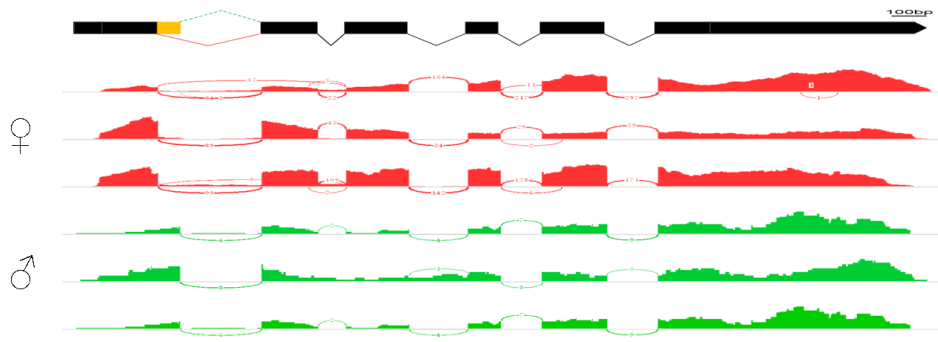

b
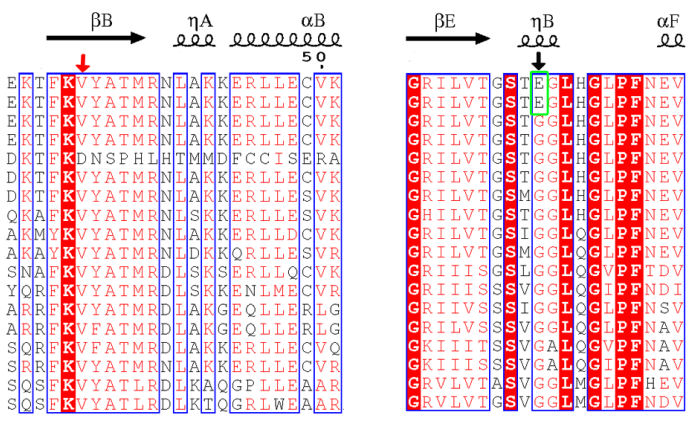

c
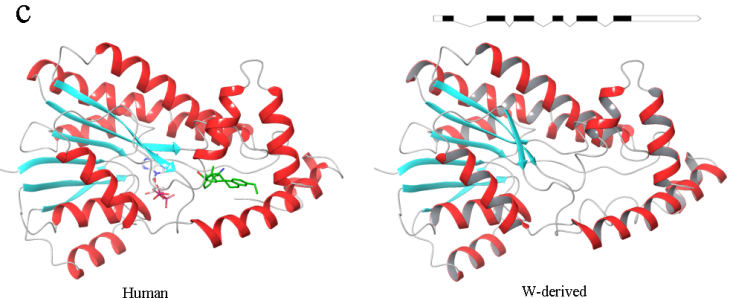

$-$

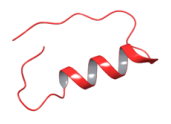

W-derived

Z-derived 
a

F0
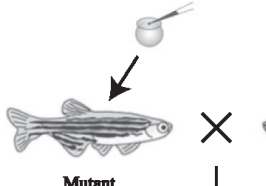

Mutant

F1
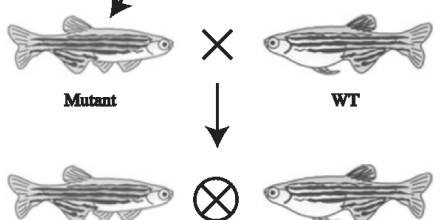

8
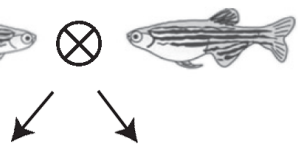

F2

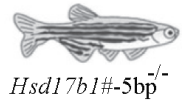

c
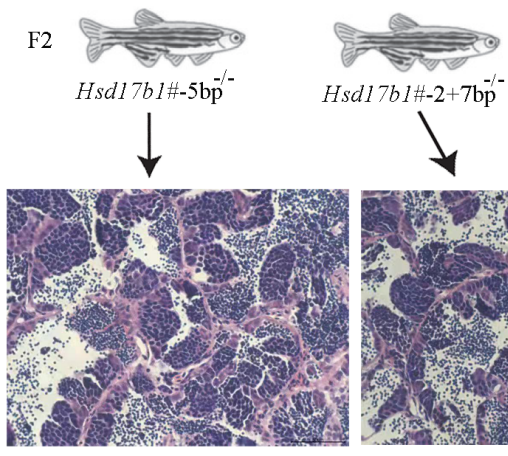

b

$H$ sal17b1

Hsd17b1 1 -5bp

Wild

$H s d 17 b 1 \#-2+7 \mathrm{bp}$

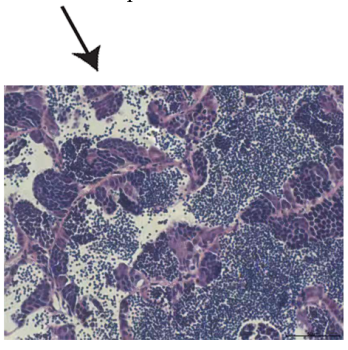

d

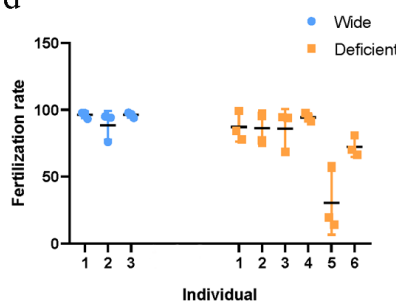

a

Chr16:18219150 Chr16:18219853 or SNP1196

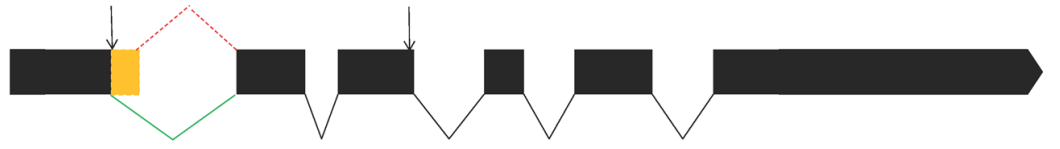

b

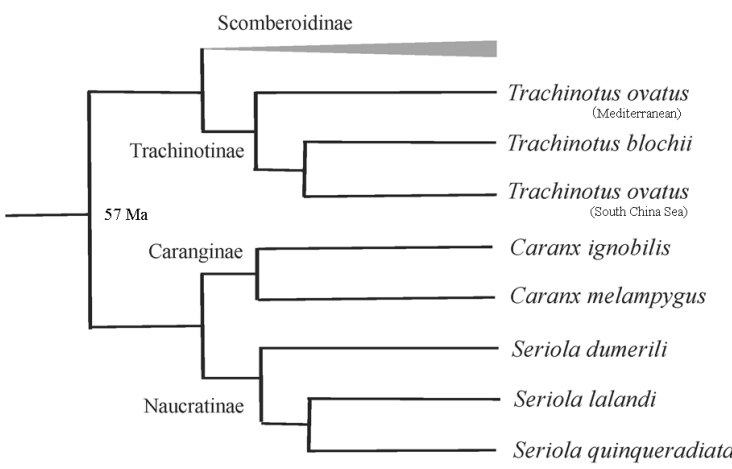

\begin{tabular}{|c|c|c|}
\hline Chr16:18219150 & SNP1196 & Gene \\
\hline$\times$ & $\times$ & $?$ \\
\hline$x$ & $\times$ & $?$ \\
\hline$v$ & $\times$ & Hsdl7bl \\
\hline$\times$ & $\times$ & $?$ \\
\hline$\times$ & $\times$ & $?$ \\
\hline$\times$ & $\sqrt{ }$ & Hsdl7b1 \\
\hline$\times$ & $\sqrt{ }$ & Hsdl7bl \\
\hline$\times$ & $\sqrt{ }$ & Hsdl7b1 \\
\hline
\end{tabular}



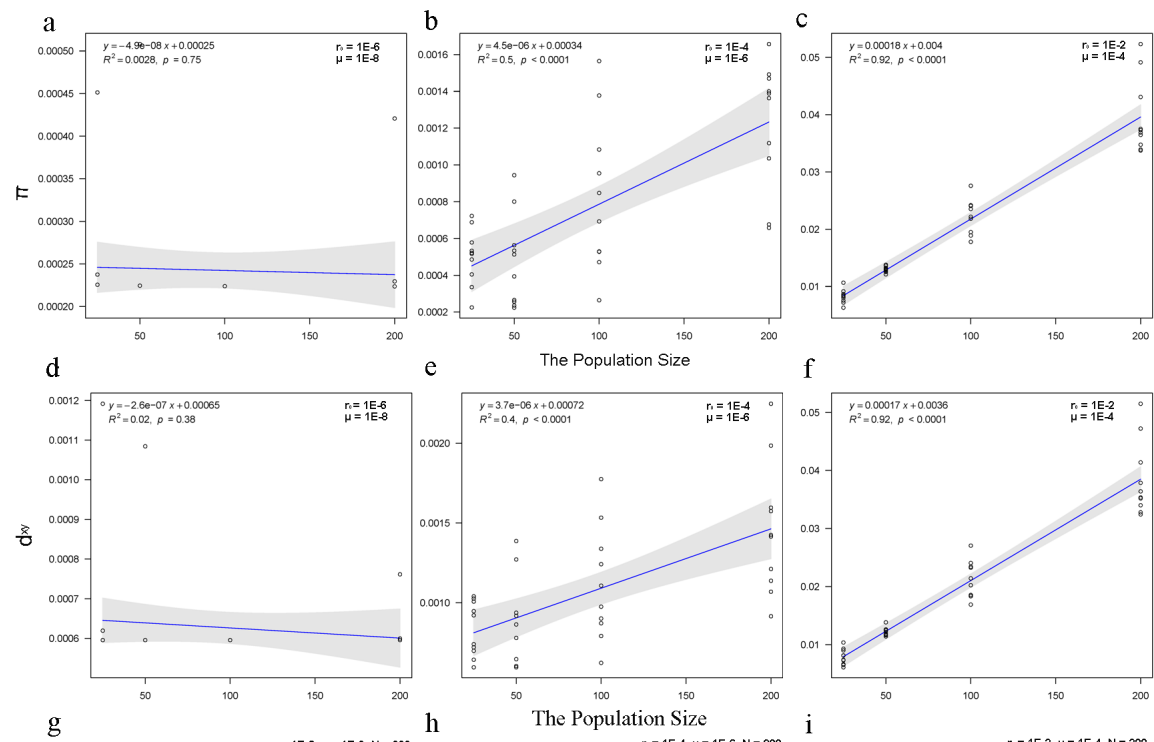

f
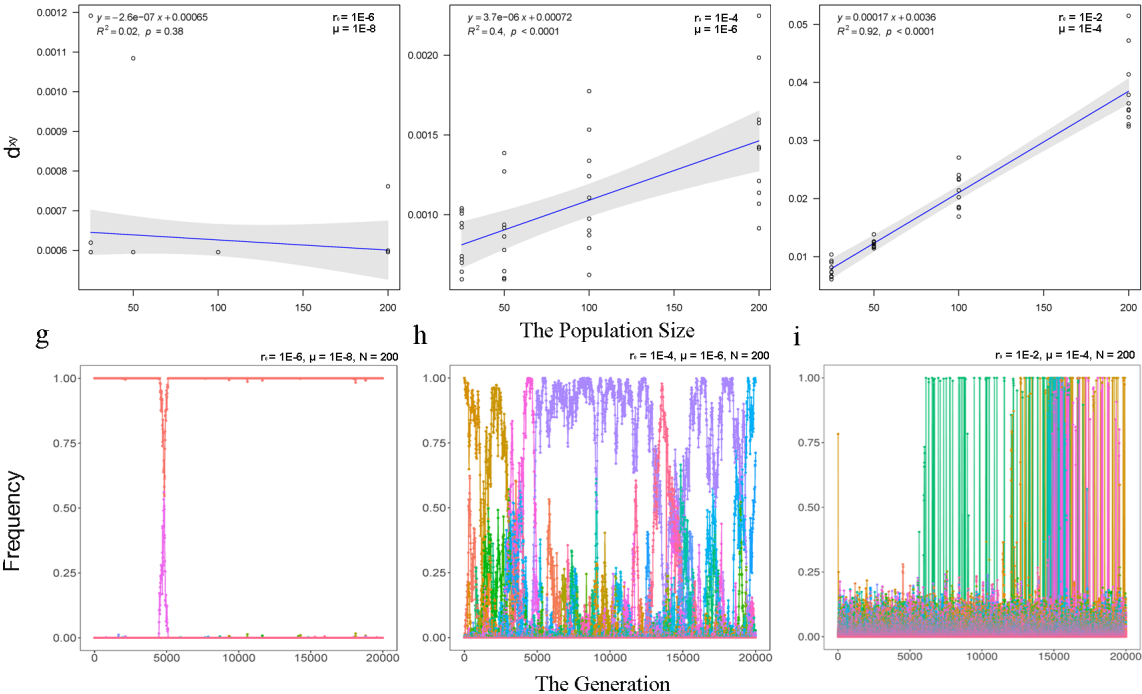